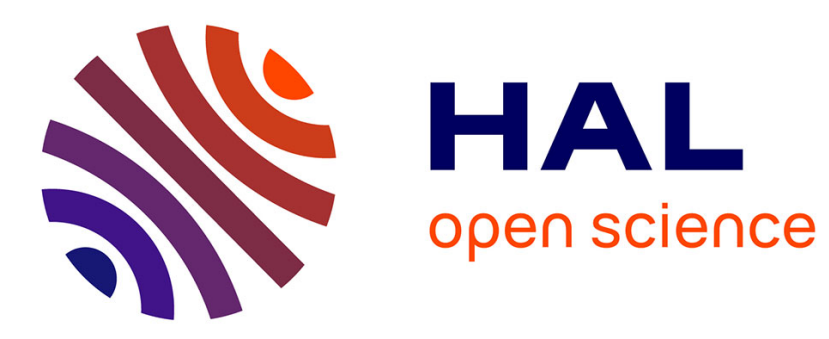

\title{
Mechanical properties and impedance model for the branching network of the sapping system in the leaf of
}

Clara Ionescu, J. Tenreiro Machado

\section{- To cite this version:}

Clara Ionescu, J. Tenreiro Machado. Mechanical properties and impedance model for the branching network of the sapping system in the leaf of . Nonlinear Dynamics, 2009, 60 (1-2), pp.207-216. 10.1007/s11071-009-9590-0 . hal-00568397

\section{HAL Id: hal-00568397 \\ https://hal.science/hal-00568397}

Submitted on 23 Feb 2011

HAL is a multi-disciplinary open access archive for the deposit and dissemination of scientific research documents, whether they are published or not. The documents may come from teaching and research institutions in France or abroad, or from public or private research centers.
L'archive ouverte pluridisciplinaire HAL, est destinée au dépôt et à la diffusion de documents scientifiques de niveau recherche, publiés ou non, émanant des établissements d'enseignement et de recherche français ou étrangers, des laboratoires publics ou privés. 
nody9590_source.doc

\title{
Mechanical properties and impedance model for the branching network of the sapping system in the leaf of Hydrangea Macrophylla
}

\author{
Clara Ionescu $^{1}, \mathrm{~J}$ Tenreiro Machado ${ }^{2}$ \\ ${ }^{1}$ Ghent University, Department of Electrical energy, Systems and Automation, \\ Technologiepark 913, B9052, Gent, Belgium \\ ${ }^{2}$ Department of Electrical Engineering, Institute of Engineering of Porto, Porto, Portugal
}

\begin{abstract}
An electrical analogue model has been developed based on main leaf hydraulics characteristics and intrinsic geometry. The simulations show good qualitative agreements with specialized literature reports. The constant-phase behavior and the variation with ambient temperature of the frequency response of the leaf impedance are assessed by means of simulation studies.
\end{abstract}

Keywords: fractal; impedance; constant-phase behavior; power-law; leaf; frequency domain; hydraulics; mechanics

\section{Nomenclature}

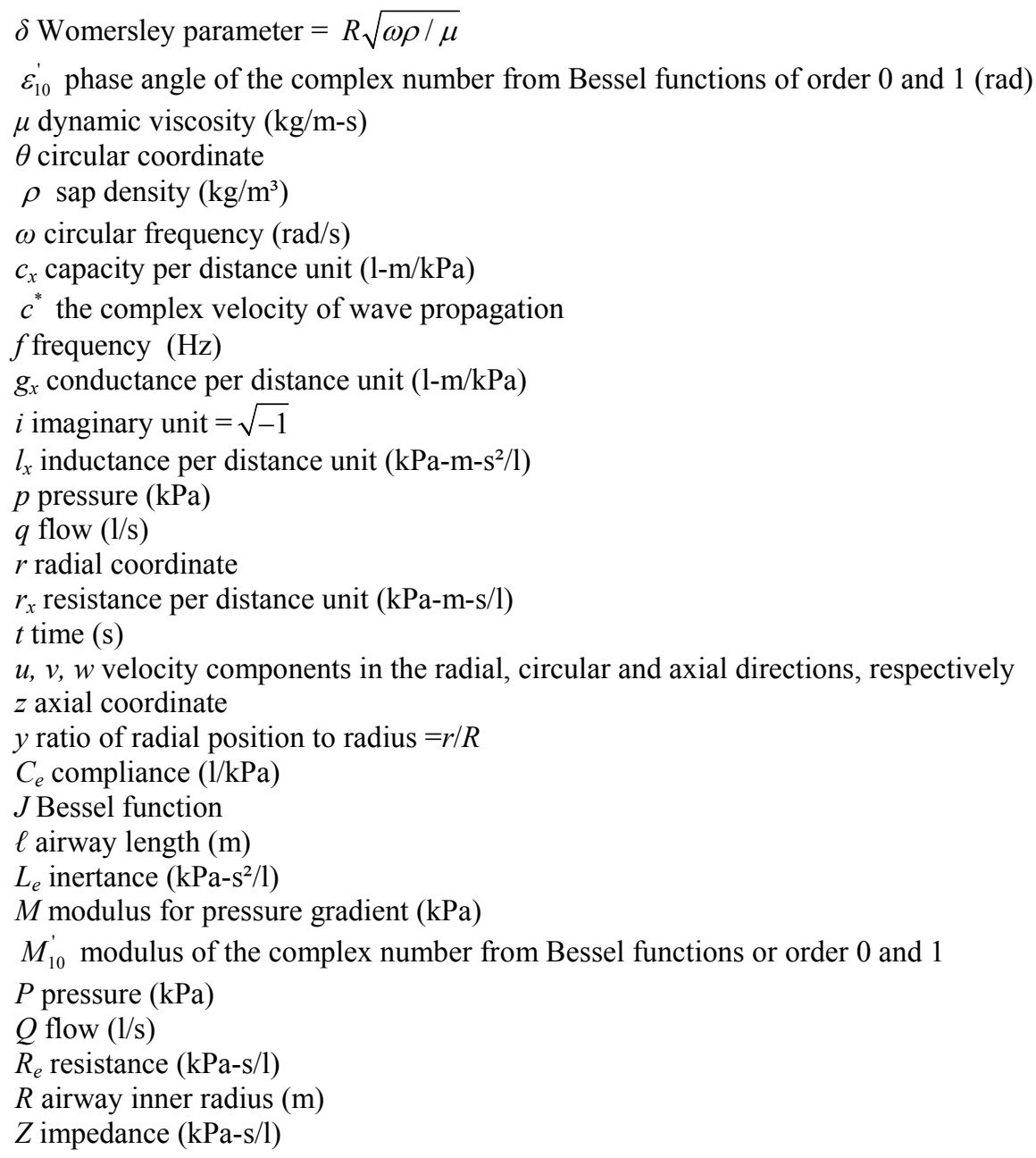


nody9590_source.doc

\section{Introduction}

Branching transport networks in biology are designed such that they are energetically optimal, a crucial condition in species survival [6]. Such networks are then constructed by vessels in a bifurcating structure, for instance the circulatory $[7,12]$ or the respiratory systems $[8,10,12]$. The relationship between the parent and daughter branch will then follow a relation as a function of a bifurcation exponent, showing the property of self-organized critically. At the network level, based on a fractal pattern, this exponent will reflect the overall fluid dynamics efficiency and energy dissipation during transport [8]. This interesting theory may be extended to other networks, such as those of sap tubes in plants, where one may also recognize the presence of the branching pattern [13].

In a plant, the leaf is metabolically very active, functioning as the site of photosynthesis, which determines the primary processes occurring within the plant. Leaves are also a major site of carbohydrate and mineral storage. Consequently, the mineral status of the leaves not only influences the efficacy of photosynthesis, but also reflects the nutrient status of the plant and therefore its fruitfulness $[14,19]$. Although other organs within the plant may act in a similar manner, the leaf is the most readily available source of tissue for analysis.

Plant biologists spent many decades trying to understand the mechanisms behind the transport of nutrients through sap tubes in the phloem $[4,5,14]$. Proponents of mass transport believed the gradient of solute concentration along sap tubes would create a gradient of turgor pressure. The pressure gradient could be quite small, namely $0.020 \mathrm{MPa}$ per meter, but still be enough to drive the flow of nutrients [14]. Therefore, one may consider laminar flow conditions and apply some well-known theoretical studies for analysis $[10,17,18]$.

In this contribution, a model is developed for the branching tubular structure of the leaf by means of its electrical impedance analogue. In order to achieve this goal, a theoretical analysis will determine the conditions in which the model is conceived. The final aim is to determine whether, or not, the branching, repetitive structure of the leaf may lead to dynamical properties of noninteger order impedance models in the frequency domain. A similar observation has been previously reported in [11], strengthening our argument in carrying this study.

Bearing these ideas in mind the structure of the paper is as follows. Section two introduces the theoretical basis of fluid dynamics and its electrical equivalent, and the description of the leaf under study. Section three presents the numerical results yielded by the proposed methodology and discusses the system dynamics. Finally, section four summarizes the main conclusions. 
nody9590_source.doc

\section{MATERIALS AND METHODS}

\subsection{The Navier-Stokes equations}

The nutrient in the leaf-tubes is treated as Newtonian, with constant viscosity $\mu=0.0015 \mathrm{~kg} / \mathrm{m}-\mathrm{s}$ and density $\rho=1027 \mathrm{~kg} / \mathrm{m}^{3}$ (saltwater). Applying the Navier-Stokes (N-S) partial differential equations of the fluid dynamical stability in polar-cylindrical coordinates system [17], we have:

$$
\begin{aligned}
& \rho\left(\frac{\partial u}{\partial t}+u \frac{\partial u}{\partial r}+\frac{v}{r} \frac{\partial u}{\partial \theta}+w \frac{\partial u}{\partial z}-\frac{v^{2}}{r}\right)= \\
& -\frac{\partial p}{\partial r}+\rho F_{r}+\mu\left[\frac{1}{r} \frac{\partial}{\partial r}\left(r \frac{\partial u}{\partial r}\right)-\frac{u}{r^{2}}+\frac{1}{r^{2}} \frac{\partial^{2} u}{\partial \theta^{2}}-\frac{2}{r^{2}} \frac{\partial v}{\partial \theta}+\frac{\partial^{2} u}{\partial z^{2}}\right]
\end{aligned}
$$

for the radial coordinate $r$, and:

$$
\begin{aligned}
& \rho\left(\frac{\partial v}{\partial t}+u \frac{\partial v}{\partial r}+\frac{v}{r} \frac{\partial v}{\partial \theta}+w \frac{\partial v}{\partial z}+\frac{u v}{r}\right)= \\
& -\frac{1}{r} \frac{\partial p}{\partial \theta}+\rho F_{\theta}+\mu\left[\frac{1}{r} \frac{\partial}{\partial r}\left(r \frac{\partial v}{\partial r}\right)-\frac{v}{r^{2}}+\frac{1}{r^{2}} \frac{\partial^{2} v}{\partial \theta^{2}}+\frac{2}{r^{2}} \frac{\partial u}{\partial \theta}+\frac{\partial^{2} v}{\partial z^{2}}\right]
\end{aligned}
$$

for the circular coordinate $\theta$ and:

$$
\begin{aligned}
& \rho\left(\frac{\partial w}{\partial t}+u \frac{\partial w}{\partial r}+\frac{v}{r} \frac{\partial w}{\partial \theta}+w \frac{\partial w}{\partial z}\right)= \\
& -\frac{\partial p}{\partial z}+\rho F_{z}+\mu\left[\frac{1}{r} \frac{\partial}{\partial r}\left(r \frac{\partial w}{\partial r}\right)+\frac{1}{r^{2}} \frac{\partial^{2} w}{\partial \theta^{2}}+\frac{\partial^{2} w}{\partial z^{2}}\right]
\end{aligned}
$$

in the axial coordinate $z$, where $u, v, w$ are Euler fluid velocity components in the radial, circular and axial directions, respectively; $p$ is the pressure and $F$ denote external forces. Due to axial symmetry of the flow, the circular component of the velocity is equal to zero, $v=0$. Similarly, the radial and the axial components of the velocity are function of the radial and axial coordinates as in: $\frac{\partial u(r, z, t)}{\partial \theta}=0, \frac{\partial^{2} u(r, z, t)}{\partial \theta^{2}}=0, \frac{\partial w(r, z, t)}{\partial \theta}=0, \frac{\partial^{2} w(r, z, t)}{\partial \theta^{2}}=0$. Considering null external forces $F$ and dividing by the density $\rho$ (we consider here only those plants whose sap in the leaf is incompressible, with low pressure drop variations, $4 \mathrm{kPa}$, that is, $4 \%$ of atmospheric pressure), we have the radial and axial components defining the momentum equations as:

$$
\begin{gathered}
\frac{\partial u}{\partial t}+u \frac{\partial u}{\partial r}+w \frac{\partial u}{\partial z}=-\frac{1}{\rho} \frac{\partial p}{\partial r}+\frac{\mu}{\rho}\left[\frac{1}{r} \frac{\partial}{\partial r}+\frac{\partial^{2} u}{\partial r^{2}}+\frac{\partial^{2} u}{\partial z^{2}}-\frac{u}{r^{2}}\right] \\
\frac{\partial w}{\partial t}+u \frac{\partial w}{\partial r}+w \frac{\partial w}{\partial z}=-\frac{1}{\rho} \frac{\partial p}{\partial z}+\frac{\mu}{\rho}\left[\frac{1}{r} \frac{\partial w}{\partial r}+\frac{\partial^{2} w}{\partial r^{2}}+\frac{\partial^{2} w}{\partial z^{2}}\right]
\end{gathered}
$$


nody9590_source.doc

From the continuity equation (mass balance) we have:

$$
\frac{\partial u}{\partial r}+\frac{u}{r}+\frac{1}{r} \frac{\partial v}{\partial \theta}+\frac{\partial w}{\partial z}=0
$$

or, for an axi-symmetric case:

$$
\frac{u}{r}+\frac{\partial u}{\partial r}+\frac{\partial w}{\partial z}=0
$$

At this moment, we defined through (4), (5) and (7) the components of the velocity, in the radial direction $u(r, z, t)$ and the axial direction $w(r, z, t)$, and the pressure of the fluid $p(r, z, t)$. The dimensionless coordinate $y=\frac{r}{R}, 0 \leq y \leq 1$, which denotes the ratio of the radial position with respect to the axis of the tube and the inner tube radius $R$ is introduced and using the relation:

$$
\frac{\partial}{\partial y}=\frac{\partial}{\partial r} \frac{\partial r}{\partial y}=R \frac{\partial}{\partial r}, \quad \frac{\partial}{\partial r}=\frac{1}{R} \frac{\partial}{\partial y}
$$

the set of three equations defined before can be re-written as:

$$
\begin{aligned}
& \frac{\partial u}{\partial t}+\frac{u}{R} \frac{\partial u}{\partial y}+w \frac{\partial u}{\partial z}=-\frac{1}{\rho R} \frac{\partial p}{\partial y}+\frac{\mu}{\rho}\left[\frac{1}{y R^{2}} \frac{\partial u}{\partial y}+\frac{1}{R^{2}} \frac{\partial^{2} u}{\partial y^{2}}+\frac{\partial^{2} u}{\partial z^{2}}-\frac{u}{R^{2} y^{2}}\right] \\
& \frac{\partial w}{\partial t}+w \frac{\partial w}{\partial z}+\frac{u}{R} \frac{\partial w}{\partial y}=-\frac{1}{\rho} \frac{\partial p}{\partial z}+\frac{\mu}{\rho}\left[\frac{1}{y R^{2}} \frac{\partial w}{\partial y}+\frac{1}{R^{2}} \frac{\partial^{2} w}{\partial y^{2}}+\frac{\partial^{2} w}{\partial z^{2}}\right] \\
& \frac{u}{R y}+\frac{1}{R} \frac{\partial u}{\partial y}+\frac{\partial w}{\partial z}=0
\end{aligned}
$$

Consider further the following simplification assumptions:

- the radial velocity component is small, as well as the ratio $u / R$ and the term in the radial direction;

- the terms $\frac{u(r, z, t)}{R}<<1$ and $\frac{\partial^{2} u(r, z, t)}{R \partial z^{2}}<<y$ in the axial direction are negligible.

Consequently, the following system results:

$$
\begin{aligned}
& \frac{\partial u}{\partial t}=-\frac{1}{\rho R} \frac{\partial p}{\partial y}+\frac{\mu}{\rho}\left[\frac{1}{y R^{2}} \frac{\partial u}{\partial y}+\frac{1}{R^{2}} \frac{\partial^{2} u}{\partial y^{2}}-\frac{u}{R^{2} y^{2}}\right] \\
& \frac{\partial w}{\partial t}=-\frac{1}{\rho} \frac{\partial p}{\partial z}+\frac{\mu}{\rho}\left[\frac{1}{y R^{2}} \frac{\partial w}{\partial y}+\frac{1}{R^{2}} \frac{\partial^{2} w}{\partial y^{2}}\right] \\
& \frac{u}{R y}+\frac{1}{R} \frac{\partial u}{\partial y}+\frac{\partial w}{\partial z}=0
\end{aligned}
$$


nody9590_source.doc

The most simple scenario is to assume a constant flow rate in a rigid pipeline (without branching airways taken into account), that is, the Poiseuille flow. In the case of fully developed, laminar flow in a rigid tube, the radial velocity is zero. Moreover, if the flow is also supposed continuous, the variations with respect to time are also zero. The continuity equation (13) reduces to $\frac{\partial w}{\partial z}=0$ and (12) becomes: $\frac{1}{\rho} \frac{\partial p}{\partial z}=\frac{\mu}{\rho}\left[\frac{1}{y R^{2}} \frac{\partial w}{\partial y}+\frac{1}{R^{2}} \frac{\partial^{2} w}{\partial y^{2}}\right]$, or equivalently $\frac{R^{2}}{\mu} \frac{\partial p}{\partial z}=\frac{1}{y} \frac{\partial}{\partial y}\left[y \frac{\partial w}{\partial y}\right]$ and the solution is given by $w(y)=A \ln (y)+B+\left(\frac{R^{2}}{\mu} \frac{\partial p}{\partial z}\right) \frac{y^{2}}{4}$, with $A$ and $B$ to be determined from boundary conditions. These conditions are defined on the tube wall for $r= \pm R, w(R, z, t)=0$ component of velocity in the axial direction are equal to zero and from $y=0$ (axis of the tube), $w(y)$ converges to a finite value. For $y= \pm 1$ (tube wall) we have $w=0$ (no slip condition), and results that $B=-\frac{R^{2}}{4 \mu} \frac{\partial p}{\partial z}$. Finally, the solution is given by:

$$
w(y)=\frac{R^{2}}{4 \mu}\left(-\frac{\partial p}{\partial z}\right)\left(1-y^{2}\right)
$$

which is the equation of a parabola with the top at $y=0$ (axis of the airway) and the maximum value given by:

$$
w_{\max }=\frac{R^{2}}{4 \mu}\left(-\frac{\partial p}{\partial z}\right)
$$

while the velocity is $w=0$ at the wall of the pipeline, corresponding to $y= \pm 1$. The total flow is obtained by:

$$
Q=\int_{0}^{1} 2 \pi y R w(y) R d y
$$

from which we have:

$$
Q=\frac{\pi R^{4}}{8 \mu}\left(-\frac{\partial p}{\partial z}\right)=\frac{\pi D^{4}}{128 \mu}\left(-\frac{\partial p}{\partial z}\right)
$$

with $D=2 R$. The averaged velocity is given by $W=\frac{Q}{\pi R^{2}}=\frac{R^{2}}{8 \mu}\left(-\frac{\partial p}{\partial z}\right)$ and is half the maximal velocity (15).

The pressure gradient along the length of a plant is related to the width of the sap tubes as well as the viscosity of the solution. We can use an equation to calculate expected pressure gradients and compare these results with those of mass transport. The Poiseuille equation assumes that the pressure gradient $d P / d \ell(\mathrm{MPa} / \mathrm{m})$, created by differences in carbon concentration between phloem cells, depends on the width of those cells and the viscosity of the solution. A useful form of this equation is: 
nody9590_source.doc

$$
\frac{d P}{d \ell}=\frac{8 \mu w}{R^{2}} 10^{-5}
$$

where $\mu$ is the viscosity of the solution (around 0.015 poise), $w$ is the axial velocity $(\mathrm{m} / \mathrm{s}$ ), and $R$ is the radius of the tube $(\mathrm{m})$.

\subsection{Oscillating laminar flow conditions}

If we consider the flow in a circular pipeline, as a result of the varying pressure-gradient, we have:

$$
-\frac{\partial p}{\partial z}=\operatorname{Re}\left[M e^{i(\omega t-\Phi)}\right]=M \cos (\omega t-\Phi)
$$

where $\omega$ is the circular frequency $(\mathrm{rad} / \mathrm{s}), f$ the frequency $(\mathrm{Hz}), M$ the modulus and $\Phi$ is the phase angle ( $\mathrm{rad}$ ) of the pressure gradient at time $t=0 ; i$ is the imaginary unit $i=\sqrt{-1}$ and $\operatorname{Re}[\ldots]$ denotes the real part of the complex function. Given the pressure gradient is periodic, it follows that also the pressure $p(y, z, t)$ and the other velocity components $u(y, z, t) ; w(y, z, t)$ will also be periodic, with the same frequency. The variables can therefore be written in a similar form as the pressure gradient, yielding:

$$
\begin{aligned}
& p(y, z, t)=p_{1}(y) e^{i \omega\left(t-\frac{z}{c^{*}}\right)} \\
& u(y, z, t)=u_{1}(y) e^{i \omega\left(t-\frac{z}{c^{*}}\right)} \\
& w(y, z, t)=w_{1}(y) e^{i \omega\left(t-\frac{z}{c^{*}}\right)}
\end{aligned}
$$

with $c^{*}$ the complex velocity of wave propagation in the axial direction, and $p_{l}(y), u_{l}(y)$ and $w_{l}(y)$, $y=r / R$ are eigen amplitude functions satisfying the boundary conditions on the tube wall $r= \pm R$, $w(R, z, t)=0$, resulting that $w_{1}(R, z, t)=0$ and $u_{1}( \pm 1)=0$. The solution will consist of the modulus as a function of the dimensionless parameter $y$, and the phase as a function of time and the longitudinal coordinate $z$. Based on previously reported work [10], further simplifications lead to the following system of differential equations:

$$
\begin{aligned}
& u=\frac{i \omega R}{\mu c^{*}}\left\{C_{1} \frac{2}{\delta i^{3 / 2}} J_{1}\left(\delta i^{3 / 2} y\right)+\frac{A_{1}}{\rho c^{*}} y\right\} e^{i \omega\left(t-\frac{z}{c^{*}}\right)} \\
& w=\left\{C_{1} J_{0}\left(\delta i^{3 / 2} y\right)+\frac{A_{1}}{\rho c^{*}}\right\} e^{i \omega\left(t-\frac{z}{c^{*}}\right)} \\
& p=A_{1} e^{i \omega\left(t-\frac{z}{c^{*}}\right)}
\end{aligned}
$$

with $\delta=R \sqrt{\omega \rho / \mu}$ the Womersley parameter. The value of $C_{1}$ results from the kinematic limit (velocity), $C_{1}=-\frac{A_{1}}{\rho c^{*}} \frac{1}{J_{0}\left(\delta i^{3 / 2}\right)}$. The pressure can be re-written as a function of the pressure 
nody9590_source.doc

difference between two points: $\quad-\frac{d p}{d z}=\frac{i \omega}{c^{*}} A_{1} e^{i \omega\left(t-\frac{z}{c^{*}}\right)}=M e^{i(\omega t-\Phi)}, \quad$ such $\quad$ that $A_{1} e^{i \omega\left(t-\frac{z}{c^{*}}\right)}=\frac{c^{*}}{\omega} M e^{i\left(\omega t-\Phi-\frac{\pi}{2}\right)}$. It follows that $(20-22)$ can be re-written in function of the pressure gradient:

$$
\begin{aligned}
u & =C_{1} \frac{i \omega R}{\delta i^{3 / 2} c^{*}} J_{1}\left(\delta i^{3 / 2} y\right) e^{i \omega\left(t-\frac{z}{c^{*}}\right)}+\frac{R y}{2 \rho c^{*}} M e^{i(\omega t-\Phi)} \\
w & =C_{1} J_{0}\left(\delta i^{3 / 2} y\right) e^{i \omega\left(t-\frac{z}{c^{*}}\right)}+\frac{M}{\omega \rho} e^{i\left(\omega t-\Phi-\frac{\pi}{2}\right)} \\
-\frac{d p}{d z} & =M e^{i(\omega t-\Phi)}
\end{aligned}
$$

The three equations $\left(20^{\prime}-22^{\prime}\right)$ form the solutions of the system of differential equations given by linearization of the N-S equations and the continuity equation in the conditions of an axisymmetrical flow; circular pipeline; negligible gravity forces.

\subsection{Analysis for rigid tubes}

To determine the value of $C_{1}$ in $\left(21^{\prime}\right)$ is necessary to employ the "no-slip" condition (velocity $w=0$ at the tube wall, thus at $y= \pm 1)[9,10]$. Introducing the value for $C_{1}$ in (21') results in the axial velocity profile:

$$
w(y)=\frac{M}{\omega \rho}\left\{1-\frac{J_{0}\left(\delta i^{3 / 2} y\right)}{J_{0}\left(\delta i^{3 / 2}\right)}\right\} e^{i\left(\omega t-\Phi-\frac{\pi}{2}\right)}
$$

with $J_{0}$ the Bessel function of first kind and order zero [1]. Using the transformations from complex values to modulus-phase values, (23) can be re-written as:

$$
w(y)=\frac{M}{\omega \rho} M^{\prime}{ }_{0}^{\prime}(y) e^{i\left(\omega t-\Phi-\frac{\pi}{2}+\varepsilon_{0}^{\prime}(y)\right)}=\frac{M R^{2}}{\mu \delta^{2}} M_{0}^{\prime}(y) e^{i\left(\omega t-\Phi-\frac{\pi}{2}+\varepsilon_{0}^{\prime}(y)\right)}
$$

and the real part of (24) is given by:

$$
w(y)=\frac{M R^{2}}{\mu \delta^{2}} M^{\prime}{ }_{0}(y) \sin \left(\omega t-\Phi+\varepsilon^{\prime}{ }_{0}(y)\right)
$$

Integrating (24) and following the same reasoning as for the velocity, we obtain that

$$
Q=\frac{\pi R^{2}}{\omega \rho}\left\{1-\frac{2 J_{1}\left(\delta i^{3 / 2}\right)}{J_{0}\left(\delta i^{3 / 2}\right) \delta i^{3 / 2}}\right\} M e^{i\left(\omega t-\Phi-\frac{\pi}{2}\right)}
$$


nody9590_source.doc

The averaged velocity can be found by dividing $Q$ to the surface, $\pi R^{2}$. In a similar fashion as for (24), the modulus and phase can be found as:

$$
Q=\frac{\pi R^{2} M}{\omega \rho} M_{10}^{\prime} e^{i\left(\omega t-\Phi-\frac{\pi}{2}+\varepsilon_{10}^{\prime}\right)}=\frac{\pi R^{4} M}{\mu \delta^{2}} M_{10}^{\prime} e^{i\left(\omega t-\Phi-\frac{\pi}{2}+\varepsilon_{10}^{\prime}\right)}
$$

or the corresponding real part given by:

$$
Q=\frac{\pi R^{2} M}{\omega \rho} M^{\prime}{ }_{10} \sin \left(\omega t-\Phi+\varepsilon_{10}^{\prime}\right)=\frac{\pi R^{4} M}{\mu \delta^{2}} M_{10}^{\prime} \sin \left(\omega t-\Phi+\varepsilon^{\prime}{ }_{10}\right)
$$

In relation to the Poiseuille-flow, for $\delta \rightarrow 0$ ( or $\omega=0$ for stationary flow) we have that $\frac{M_{10}^{\prime}}{\delta^{2}}=\frac{1}{8}$ and $\varepsilon_{10}^{\prime}=90^{\circ}$, such that (28) becomes $Q=\frac{\pi(2 R)^{4}}{128 \mu} M \cos (\omega t-\Phi)$ or $Q=\frac{\pi(2 R)^{4}}{128 \mu} \frac{\Delta p}{\ell}$, with $\Delta p$ the pressure gradient and $\ell$ the length of the tube. In case of the leaf tubes, we always have $\delta<1$, thus the simplifying assumptions are acceptable.
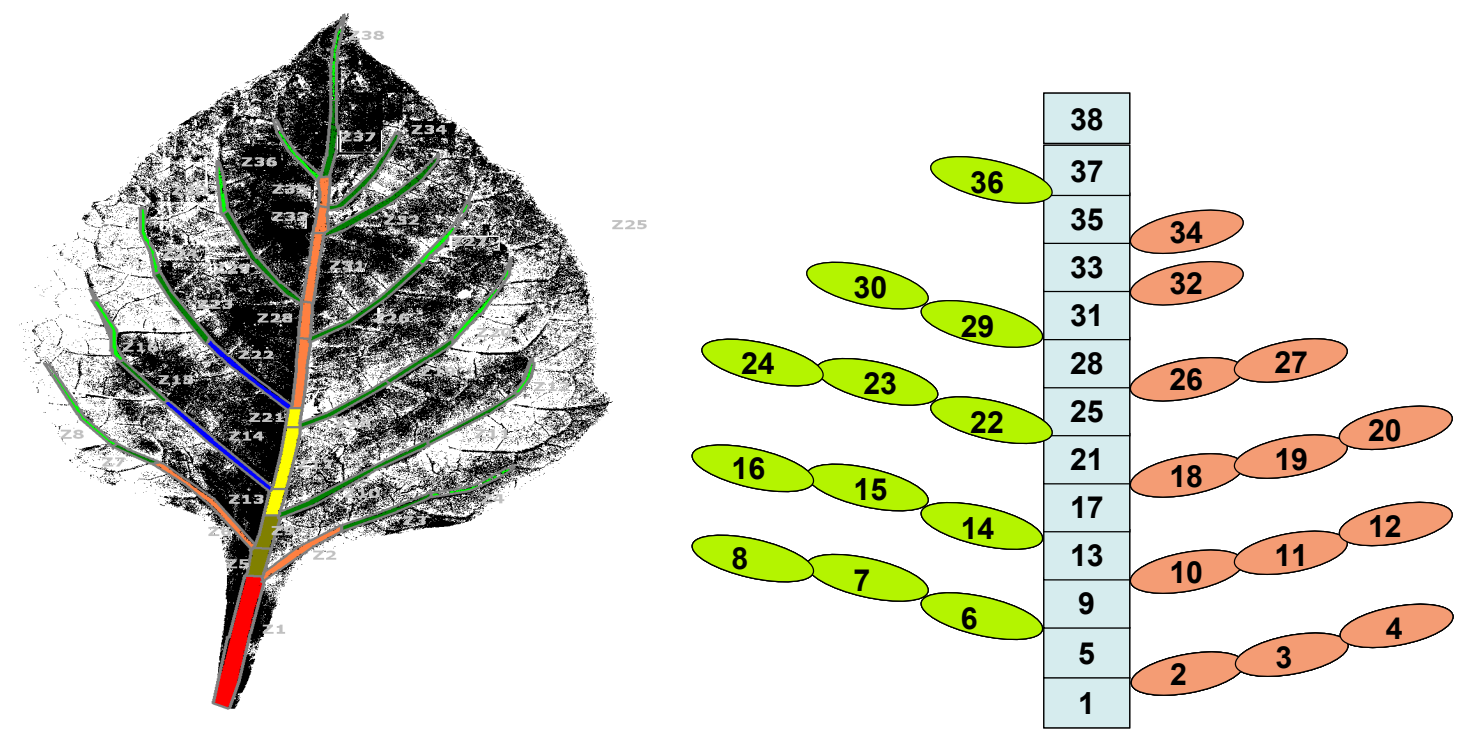

Figure 1: photocopy of the leaf under analysis (left) and notations for each branch in terms of its impedance, from 1 to 38 (right). 
nody9590_source.doc

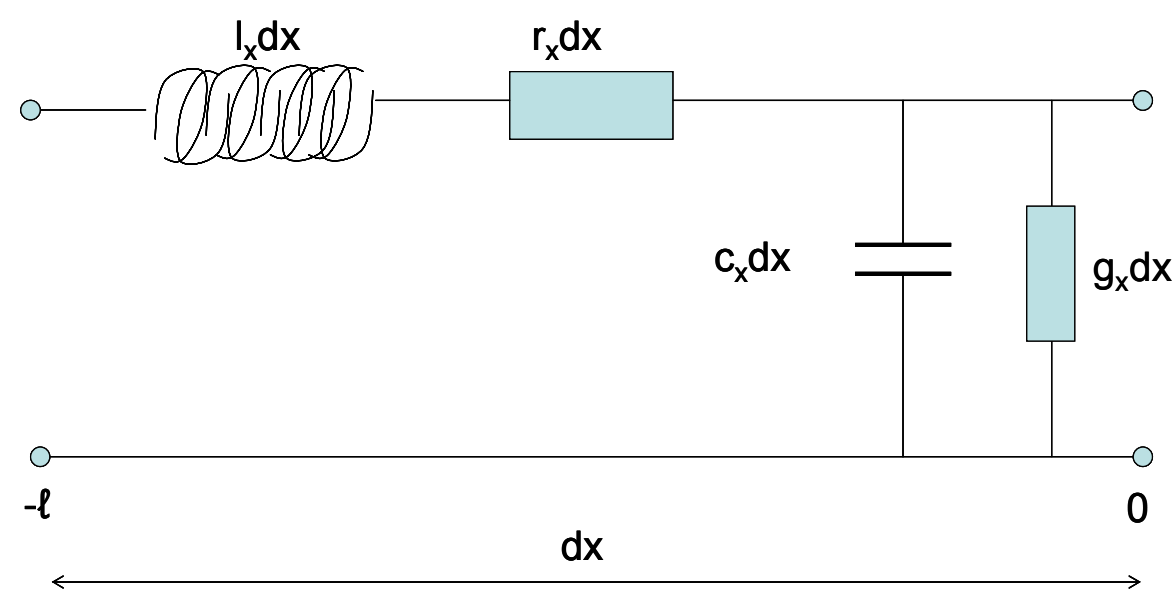

Figure 2: Infinitesimal distance $d x$ over the general representation of the transmission line

\subsection{Electrical analogue model}

Suppose the infinitesimal distance $d x$ of a transmission line as depicted in figure 2 [16]. We have the distance-dependent parameters: $l_{x}$ (induction $\left./ \mathrm{m}\right), r_{x}$ (resistance $\left./ \mathrm{m}\right), g_{x}$ (conductance $\left./ \mathrm{m}\right)$ and $c_{x}$ (capacity/m). These elements are modeled as discrete resistance $\left(r_{x} d x\right)$, conductance $\left(g_{x} d x\right)$, induction $\left(l_{x} d x\right)$ and capacity $\left(c_{x} d x\right)$. In the present study, we do not consider conductance or capacity parameters. 
nody9590_source.doc

Table 1: Measured values from the photocopied leaf structure for Hydrangea Macrophylla, for the tube radius and length. Notice the colors matching with the schematic overview from figure 1. One may observe the fact that central tubes tend to have the same ratio, whereas a similar observation ca be made for the left and right tubes.

\begin{tabular}{|c|c|c|c|c|c|c|c|}
\hline TUBE & $\mathbf{Z}$ & Radius $(m)^{*} 10 \mathrm{e}-6$ & Radius ratio & Radius ratio & Length $(m) * 10 e-6$ & Length ratio & Length ratio \\
\hline Level 1 center & 1 & 0,25 & & & 0,05 & & \\
\hline right & 2 & 0,125 & & & 0,025 & & \\
\hline right & 3 & 0,1 & 0,8 & & 0,025 & 1,00 & \\
\hline right & 4 & 0,075 & 0,75 & & 0,025 & 1,00 & \\
\hline Level 1 center & 5 & 0,225 & & 0,90 & 0,01 & & 0,20 \\
\hline Level 2 left & 6 & 0,1 & & & 0,045 & & \\
\hline Level 3 left & 7 & 0,1 & 1 & & 0,015 & 0,33 & \\
\hline Level 4 left & 8 & 0,075 & 0,75 & & 0,035 & 2,33 & \\
\hline Level 1 center & 9 & 0,225 & & 1,00 & 0,015 & & 1,50 \\
\hline Level 2 right & 10 & 0,125 & & & 0,04 & & \\
\hline Level 3 right & 11 & 0,1 & 0,8 & & 0,02 & 0,50 & \\
\hline Level 4 right & 12 & 0,075 & 0,75 & & 0,01 & 0,50 & \\
\hline Level 1 center & 13 & 0,2 & & 0,89 & 0,01 & & 0,67 \\
\hline Level 2 left & 14 & 0,1 & & & 0,05 & & \\
\hline Level 3 left & 15 & 0,1 & 1 & & 0,015 & 0,30 & \\
\hline Level 4 left & 16 & 0,075 & 0,75 & & 0,025 & 1,67 & \\
\hline Level 1 center & 17 & 0,2 & & 1,00 & 0,03 & & 3,00 \\
\hline Level 2 right & 18 & 0,125 & & & 0,03 & & \\
\hline Level 3 right & 19 & 0,1 & 0,8 & & 0,025 & 0,83 & \\
\hline Level 4 right & 20 & 0,075 & 0,75 & & 0,035 & 1,40 & \\
\hline Level 1 center & 21 & 0,175 & & 0,88 & 0,01 & & 0,33 \\
\hline Level 2 left & 22 & 0,1 & & & 0,04 & & \\
\hline Level 3 left & 23 & 0,075 & 0,75 & & 0,025 & 0,63 & \\
\hline Level $4 \quad$ left & 24 & 0,05 & 0,666666667 & & 0,03 & 1,20 & \\
\hline Level 1 center & 25 & 0,15 & & 0,86 & 0,03 & & 3,00 \\
\hline Level 2 right & 26 & 0,1 & & & 0,055 & & \\
\hline Level 3 right & 27 & 0,05 & 0,5 & & 0,015 & 0,27 & \\
\hline Level 1 center & 28 & 0,125 & & 0,83 & 0,02 & & 0,67 \\
\hline Level 2 left & 29 & 0,1 & & & 0,03 & & \\
\hline Level 3 left & 30 & 0,1 & 1 & & 0,015 & 0,50 & \\
\hline Level 1 center & 31 & 0,125 & & 1,00 & 0,025 & & 1,25 \\
\hline Level 2 right & 32 & 0,125 & & & 0,035 & & \\
\hline Level 1 center & 33 & 0,125 & & 1,00 & 0,01 & & 0,40 \\
\hline Level 2 right & 34 & 0,1 & & & 0,03 & & \\
\hline Level 1 center & 35 & 0,125 & & 1,00 & 0,012 & & 1,20 \\
\hline Level 2 left & 36 & 0,075 & & & 0,025 & & \\
\hline Level 2 center & 37 & 0,075 & & & 0,03 & & 2,50 \\
\hline Level 3 center & 38 & 0,05 & & & 0,025 & & 0,83 \\
\hline
\end{tabular}

We consider the analogy: the voltage represents the pressure $p(x, t)$, the current represents the sapflow $q(x, t)$ and we apply the transmission line theory $[3,10]$. We shall make use of the complex notation:

$$
p(x, t)=P(x) e^{i\left(\omega t-\varphi_{P}\right)}, q(x, t)=Q(x) e^{i\left(\omega t-\varphi_{Q}\right)}
$$


nody9590_source.doc

where $x$ is the longitudinal coordinate $(\mathrm{m}), t$ is the time (s), and $\omega=2 \pi f$ is the angular frequency $\left(\mathrm{rads}^{-1}\right)$. The pressure difference between two points on the transmission line denoted as $x$ and $x d x$ is due to losses over the resistance and inductance, as given by:

$$
p(x+d x)-p(x)=-r_{x} d x \cdot q-l_{x} d x \frac{\partial q}{\partial t}
$$

After division with $d x$ (assumed to be constant) and knowing that in the limit $d x \rightarrow 0$, we have that:

$$
\begin{aligned}
& \frac{\partial p}{\partial x}=-r_{x} q-l_{x} \frac{\partial q}{\partial t} \\
& \frac{\partial^{2} p}{\partial x^{2}}=-r_{x} \frac{\partial q}{\partial x}-l_{x} \frac{\partial^{2} q}{\partial x \partial t}
\end{aligned}
$$

Introducing (29) in (31) we obtain respectively:

$$
\begin{aligned}
& \frac{\partial P}{\partial x}=-\left(r_{x}+i \omega l_{x}\right) Q=-Z Q \\
& \frac{\partial^{2} P}{\partial x^{2}}=-\left(r_{x}+i \omega l_{x}\right) \frac{\partial Q}{\partial x}=-Z \frac{\partial Q}{\partial x}
\end{aligned}
$$

From these equations we have that the tube impedance is given by $Z=\frac{-\frac{\partial P}{\partial x}}{Q}=r_{x}+i \omega l_{x}$. From and (27) we have the following relation for the impedance:

$$
Z=\frac{i \omega \rho}{\pi R^{2} M_{10}^{\prime}} e^{-i \varepsilon_{10}^{\prime}}=\frac{\mu \delta^{2}}{\pi R^{4} M_{10}^{\prime}} e^{-i\left(\frac{\pi}{2}-\varepsilon_{10}^{\prime}\right)}=\frac{\mu \delta^{2}}{\pi R^{4} M_{10}^{\prime}} \sin \left(\varepsilon_{10}^{\prime}\right)+i \cos \left(\varepsilon_{10}^{\prime}\right)
$$

and by equivalence of the two relations we have that:

$$
r_{x}=\frac{\mu \delta^{2}}{\pi R^{4} M_{10}^{\prime}} \sin \left(\varepsilon_{10}^{\prime}\right)
$$

and $\omega l_{x}=\frac{\mu \delta^{2}}{\pi R^{4} M_{10}^{\prime}} \cos \left(\varepsilon_{10}^{\prime}\right)$, recalling that $\delta=R \sqrt{\frac{\omega \rho}{\mu}}$ we obtain that:

$$
l_{x}=\frac{\rho}{\pi R^{2}} \frac{\cos \left(\varepsilon_{10}^{\prime}\right)}{M_{10}^{\prime}}
$$

One can estimate that over the length $\ell$ of an airway tube (thus $x=\ell$ ), we have the corresponding properties:

$$
\begin{aligned}
& R_{e}=r_{x} \ell=\ell \frac{\mu \delta^{2}}{\pi R^{4} M_{10}^{\prime}} \sin \left(\varepsilon_{10}^{\prime}\right) \\
& L_{e}=l_{x} \ell=\ell \frac{\rho}{\pi R^{2}} \frac{\cos \left(\varepsilon_{10}^{\prime}\right)}{M_{10}^{\prime}}
\end{aligned}
$$

with values from Table 1, corresponding for each tube segment. 
nody9590_source.doc

\section{RESULTS AND DISCUSSION}

The viscosity-temperature relation can be expressed as a power law function, whereas $\mu=A e^{E v / k T}$ with $v$ the kinematic viscosity, $k$ Boltzmann's constant, $T$ the temperature, and $A$ and $E$ arbitrary constants $[4,5,19]$. The variations we have considered from literature are given in table 2 .

Table 2: Variations in the viscosity parameter with ambient temperature, as from $[5,19]$.

\begin{tabular}{|c|c|}
\hline Temperature $\left({ }^{\circ} \mathbf{C}\right)$ & Viscosity $(\mathbf{k g ~ m} / \mathbf{s})$ \\
\hline 0 & 0.014 \\
\hline 15 & 0.040 \\
\hline 20 & 0.150 \\
\hline 30 & 0.610 \\
\hline 35 & 0.820 \\
\hline
\end{tabular}
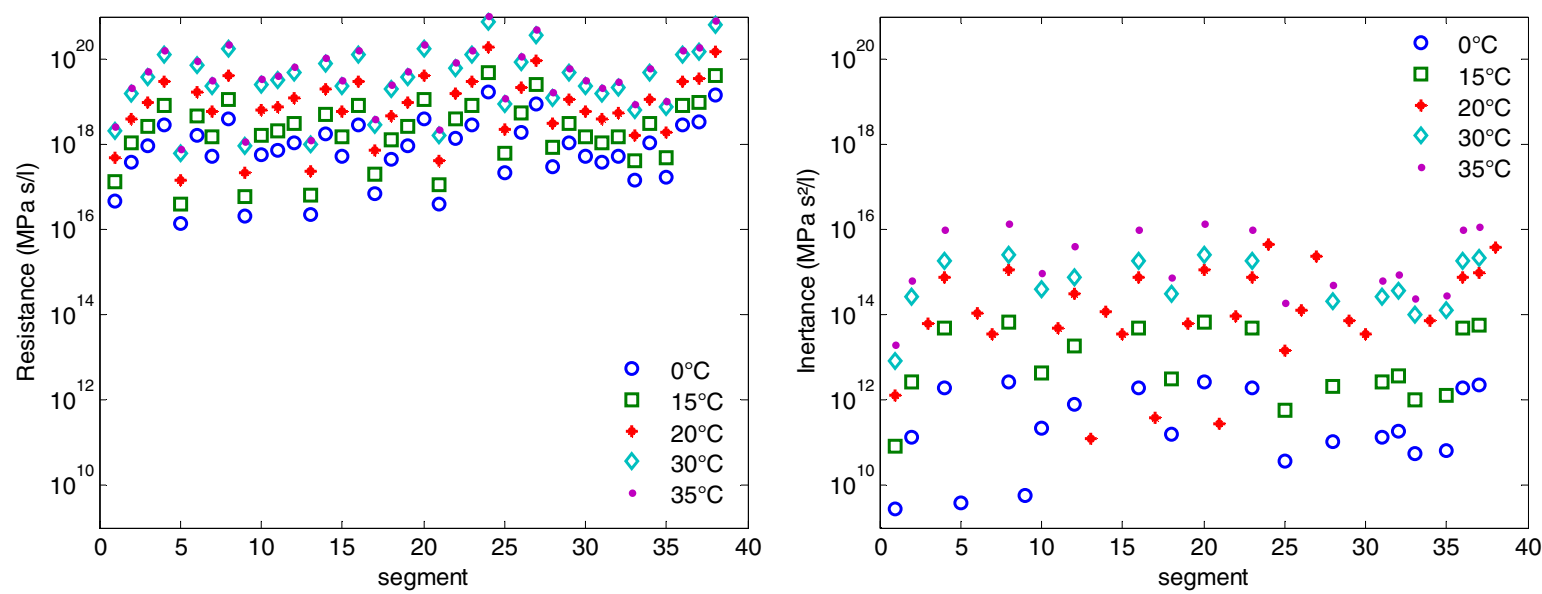

Figure 3: the variations in the mechanical parameters: resistance and inertance, with each tube segment and with temperature (via viscosity parameter). 
nody9590_source.doc
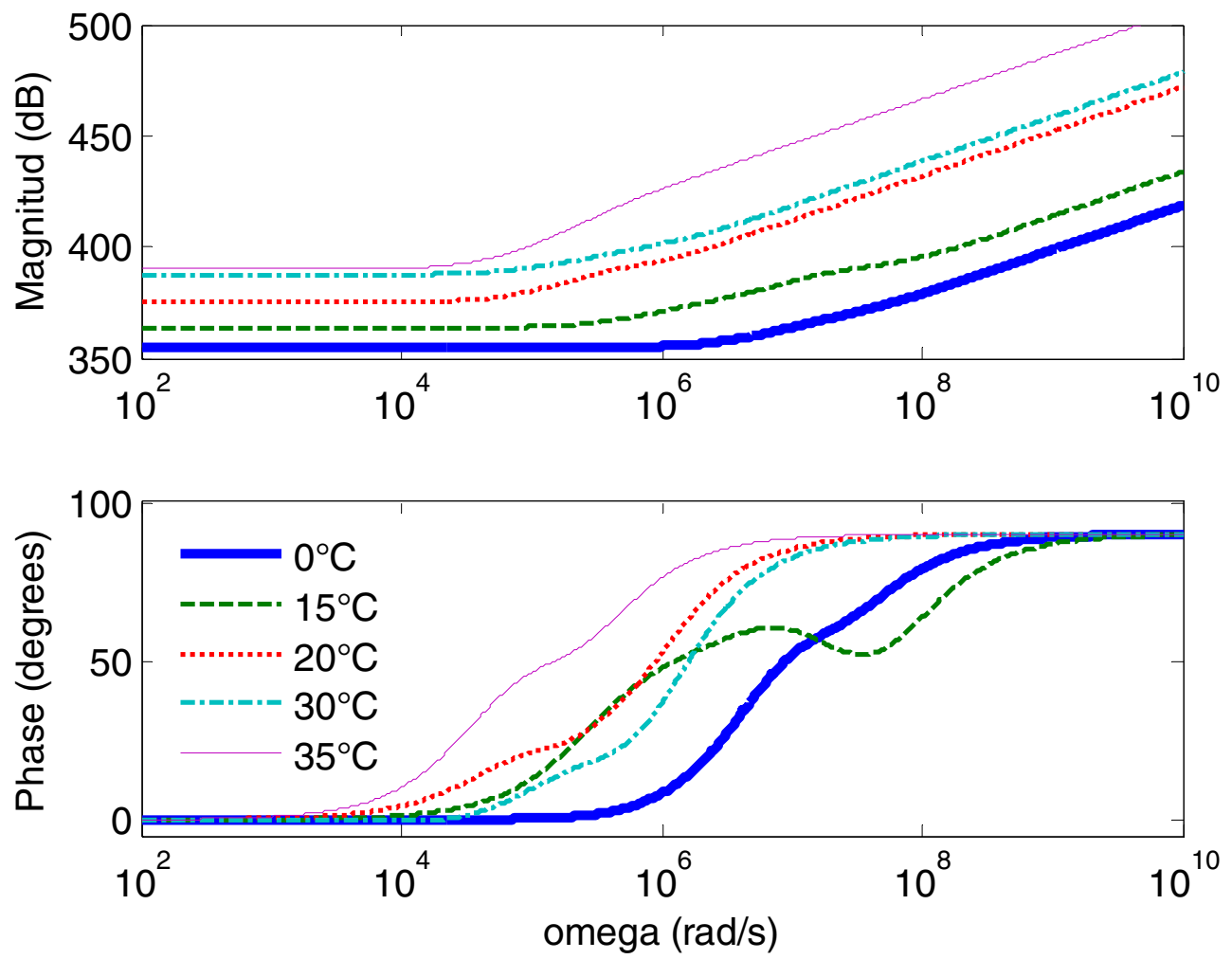

Figure 4: Bode plot for the leaf input impedance, depending on temperature (via viscosity parameter): for $0^{\circ} \mathrm{C}$ (continuous bolded line); $15^{\circ} \mathrm{C}$ (dashed line); $20^{\circ} \mathrm{C}$ (dotted line); $30^{\circ} \mathrm{C}$ (dashdotted line) and $35^{\circ} \mathrm{C}$ (continuous thin line).

The variation of the mechanical parameters resistance and inductance within consequent branches, with ambient temperature (via the viscosity parameter) is depicted in figure 3 . It can be observed that the resistance increases within the branches of consecutive levels, whereas remains constant within branches of same level. This can be done by relating figure 3 with data from Table 1 . The variability of the resistance values (standard deviations from the mean values) decreases with temperature. The mass inertia, denoted by inertance, increases with temperature, suggesting that more nutrient intake is performed at higher temperatures. This is in accordance with other studies, on total leaf conductance, supporting the idea of leaf activity with ambient temperature (e.g., photosynthesis, water content).

An important element in the leaf impedance is the presence of stomata, which allow photosynthesis by gas exchange and diffusion, a concept quite similar to the human respiratory system $[8,10]$. The gas exchange can be viewed as an elastic loss, modeled thus by a capacitance $\mathrm{C}$ conductance $\mathrm{G}$ in parallel with the series RL element. However, in order to compute the values for the $\mathrm{G}$ parameter, one needs the complex elastic modulus (i.e., bulk and shear modulus). The elastic modulus will depend on the presence of turgor and water content in the tissue cells, which in turn depends on environmental conditions [15]. Since the values vary significantly in function of the lignum percent in the leaf, which varies also with the species, it is important that one quantifies the correct value when simulation studies are intended. In this preliminary study, due to the 
nody9590_source.doc

unavailability of rheological data for our species Hydrangea Macrophylla, we will limit our model to the RL series impedance. The modeling errors introduced by the absence of capacitance or conductance parameters, are corrected by assuming a loss in the impedance of a tube of 10 percent. The reason for this consideration is twofold: $i$ ) the inter-connective tissue between the main tubes, such as those visible in figure 1 , and $i$ ) the transpiration process of the leaf. The inter-connective tissue will account for some shunt effect, given that part of the sap from the main tubes (denoted by Z1 to Z38) will be trapped between the main streams. The transpiration process will then be directly related to some losses present in each impedance term. Therefore, we assume a 10 percent loss from the tube impedance as due to these effects $[4,14]$.

The simulation of the total input impedance is done in function of all the other impedances; i.e. the total input impedance (thus at the beginning of the networking) is Z1. In figure 4, we have plotted the input impedances for various values of viscosity as a function of the ambient temperature. It is interesting to observe the merely constant-phase behavior, suggesting a relation between the fractal geometry of the leaf network of sap tubes and the frequency response. It is also clear that the frequency response varies as a (nonlinear) function of the viscosity, which in turn depends in a power law relation on temperature. Our conclusion is in agreement with previous studies involving frequency response of leaf conductance, revealing a quasi-linear dependence with temperature $[4,5]$. The constant-phase behavior occurs within a limited ambient temperature interval, i.e. at $0^{\circ} \mathrm{C}$ and $35^{\circ} \mathrm{C}$ is almost non-existent, while it is most pronounced at $15^{\circ} \mathrm{C}$. The values of the phase constancy changes as well, from about $60^{\circ}$ phase at $15^{\circ} \mathrm{C}$ to about $30^{\circ}$ phase at $20^{\circ} \mathrm{C}$ and $30^{\circ} \mathrm{C}$. This would indirectly suggest some optimality condition for the leaf sap exchange in function of the ambient conditions (here, ambient temperature).

A previous study involving frequency domain analysis of leaves over wide frequency range assumes a resistance in parallel with a capacitance, but takes not into account the nonlinear dependence with temperature (via the viscosity parameter) [2]. Although the authors acknowledge the presence of a fractional order dependence on frequency, they do not refer to the constant-phase behavior in Bode plot equivalent, but in complex impedance representation. Moreover, their observations are originated by dielectric properties, from dielectric spectroscopy measures, and not from intrinsic geometrical values.

The original contribution of this study is therefore the assessment of a constant-phase behavior arising from the intrinsic geometry of the leaf network of sap tubes, and a preliminary electrical ladder network equivalent of basic leaf hydraulics. The main limitation of the present model is that it does not incorporate the diffusion and transpiration phenomena involved in the photosynthesis. Also, elasticity of the leaf tissue is neglected, thus assuming rigid tubes.

Although simplified, the model we present here can be of great interest to study the total impedance of the plat under fractal geometry conditions. It has been recently reported that the morphology of the plant (thus including the leaf itself) can be expressed as allometric relationships, characterized by power laws; the latter being directly related to the appearance of fractional order impedance [13]. Under certain optimal conditions (e.g., symmetry and homogeneity) the hydraulic resistance to flow in xylem elements can be described by the Poiseuille equation $Q=\frac{\pi(2 R)^{4}}{128 \mu} \frac{\Delta p}{\ell}$. This implies that if the viscosity is constant, resistance will increase linearly with the tube length, 
nody9590_source.doc

but inversely with a power of the radius. The authors from [13], show that an allometric relation for whole-plant morphology and mass exist. This then would suggest the presence of fractal geometry and fractal orders of impedance, which is then in agreement with our results.

\section{CONCLUSIONS}

An electrical analogue model has been developed based on main leaf hydraulics characteristics and intrinsic geometry. The simulations show good qualitative agreements with specialized literature reports. The constant-phase behavior and the variation with ambient temperature of the frequency response of the leaf impedance are assessed by means of simulation studies.

In fact, while the frequency response of the hydraulic model has visible variation with the ambient temperature, it reveals a constant-phase behavior at ambient temperatures which are compatible with optimal conditions for the leaf and in general, for the plant itself. The model presented here characterizes one leaf, which can be viewed as the equivalent of an element in a system, i.e. plant parenchyma. The global dynamics of the plant, including the roots and several layers of leaves, need to be further evaluated. The authors believe that the phase-constancy characteristic will play a key role in the overall dynamics of the plant's input impedance. A next step in completing the model is the inclusion of the specific rheological properties for the Hydrangea Macrophylla, such as complex elastic modulus and lignum percent. The final objective is to provide insight in constant-phase behavior with fractal structure of the entire plant.

\section{Acknowledgement:}

The authors acknowledge the support offered by Dr. Nuno Ferreira in measuring geometrical values.

\section{References}

1. M. Abramowitz, I.A Stegun, Handbook of Mathematical Functions with Formulas, Graphs, and Mathematical Tables, New York: Dover Publications, ISBN 978-0-486-61272-0, (1972)

2. N. Bano, A. Hashmi, "Electrical studies of leaves over wide frequency range", IEEE Trans Dielectrics and Electrical Insulation, 3(2), 229-232, (1996)

3. A. R. Bergen, V. Vittal, Power system analysis, Second Edition, Pearson Education, United States of America (1999)

4. H. Cochard, R. Martin, P. Gross, M. Bogeat-Triboulot, "Temperature effects on hydraulic conductance and water relations of Quercus robur", Journal of Experimental Botany, 51(348), 1255-1259, (2000)

5. H. Cochard, J. Venisse, T. Barigah, N. Brunel, S. Herbette, A. Guilliot, M. Tyree, S. Sakr, "Putative role of aquaporins in variable hydraulic conductance of leaves in response to light", Plant Physiology, 143, 122-133, (2007) 
nody9590_source.doc

6. A. Eke, P. Herman, L. Kocsis, L. Kozak, "Fractal characterization of complexity in temporal physiological signals", Physiol Meas, 23, R1-R38, (2002)

7. E. Gabrys, M. Rybaczuk, A. Kedzia, "Fractal models of circulatory system. Symmetrical and asymmetrical approach comparison", Chaos,Solitons and Fractals, 24(3): 707-715, (2004)

8. S. Gheorghiu, S. Kjelstrup, P. Pfeifer, M.O. Coppens, "Is the lung an optimal gas exchanger?" in Fractals in Biology and Medicine, vol IV, Eds: Losa G., Merlini D., Nonnenmacher T., Weibel E.R., Berlin: Birkhaser, 31-42, (2005)

9. H. Franken, J. Clément, M. Cauberghs, K. Van de Woestijne, "Oscillating flow of a viscous compressible fluid through a rigid tube: a theoretical model", IEEE Trans Biomed Eng, 28(5): 416-420, (1981)

10. C. Ionescu, P. Segers, R. De Keyser, (in press) "Mechanical properties of the respiratory system derived from morphologic insight", IEEE Transactions on Biomedical Engineering, DOI 10.1109/TBME.2008.2007807, (April 2009)

11. I. Jesus, T. Machado, B. Cunha, (2008) "Fractional electrical impedances in botanical elements", $J$ of Vibration and Control, special issue on "Fractional differentiation and its applications", 14, 1389-1402

12. B. Mandelbrot, The fractal geometry of nature, NY: Freeman $\& C o,(1983)$

13. C. Price, B. Enquist, V. Savage, "A general model for allometric covariation in botanical form and function", PNAS, 104(32), 13204-13209, (2007)

14. L. Sack, M. Holbrook, "Leaf Hydraulics", Annual Review Plant Biology, 57, 361-381, (2006)

15. T. Saito, I. Terashima, "Reversible decreases in the bulk elastic modulus of mature leaves of deciduous Quercus species subjected to two drought treatments", Plant, Cell and Environment, 27, 863-875, (2004)

16. J. A. Tenreiro Machado, Isabel S. Jesus, A Suggestion from the Past?, FCAA - Journal of Fractional Calculus \& Applied Analysis, vol. 7, n. 4, pp. 403-407, 2004.

17. J. Welty, C. Wicks, R. Wilson, Fundamentals of momentum, heat and mass transfer, John Wiley \& Sons, USA, (1969)

18. J. R Womersley, "An elastic tube theory of pulse transmission and oscillatory flow in mammalian arteries". Wright Air Development Center, Technical Report WADC-TR56614, (1957)

19. E. Yamazaki, O. Kurita, Y. Matsumura, "High viscosity of hydrocolloid from leaves of cochorus olitorius L.”, Food Hydrocolloids, 23(3), 655-660, (2009) 